\title{
Ezrin-radixin-moesin-binding phosphoprotein-50 regulates EGF-induced AKT activation through interaction with EGFR and PTEN
}

\author{
JUNFANG ZHENG $^{1,2}$, YUANPING DAI $^{1}$, ZHIYU YANG $^{1}$, LONGYAN YANG $^{1}$, \\ ZHIQIANG PENG ${ }^{1}$, RAN MENG ${ }^{1}$, YING XIONG ${ }^{1,2}$ and JUNQI HE ${ }^{1,2}$ \\ ${ }^{1}$ Department of Biochemistry and Molecular Biology, Capital Medical University, Beijing 100069; \\ ${ }^{2}$ Beijing Key Laboratory for Tumor Invasion and Metastasis, Cancer Institute of \\ Capital Medical University, Beijing 100069, P.R. China
}

Received July 23, 2015; Accepted September 4, 2015

DOI: 10.3892/or.2015.4375

\begin{abstract}
Dysregulated epidermal growth factor receptor (EGFR) signaling, especially EGFR/AKT signaling, plays important roles in tumorigenesis and progression, the study on intracellular regulation of this signaling pathway has great clinical significance. Phosphatase and tensin homolog deleted on chromosome ten (PTEN) is an important antagonist of AKT activity. Its regulation of AKT activity can be enhanced by ezrin-radixin-moesin-binding phosphoprotein-50 (EBP50)-mediated PTEN/EBP50/platelet-derived growth factor receptor (PDGFR) complex. EBP50 was reported to bind to EGFR, and that it may also mediate the formation of PTEN/EGFR complex to regulate EGFR/AKT signaling. In this study, experiments were performed to verify the hypothesis. Results showed that PTEN co-immunoprecipitated with EGFR, demonstrating PTEN/EGFR complex can form in tissue. Further studies showed that EBP50 knockdown decreased the amount of PTEN/EGFR complex by GST pulldown assay, and EBP50 overexpression increased the amount of PTEN/EGFR complex in a dose-dependent manner. While PTEN mutant (V403A), which can not bind with EBP50, only slightly mediated the formation of PTEN/EGFR complex,
\end{abstract}

Correspondence to: Professor Junqi He, Department of Biochemistry and Molecular Biology, Capital Medical University, 10 Xitoutiao, You An Men, Beijing 100069, P.R. China

E-mail: jq_he@ccmu.edu.cn

Abbreviations: EGFR, epidermal growth factor receptor; PTEN, phosphatase and tensin homolog deleted on chromosome ten; EBP50, ezrin-radixin-moesin-binding phosphoprotein-50; PDGFR, platelet-derived growth factor receptor; PI3K/AKT, phosphoinositide 3-kinase/AKT; MAPK, mitogen-activated protein kinase; STAT, signal transducer and activator of transcription

Key words: ezrin-radixin-moesin-binding phosphoprotein-50, phosphatase and tensin homolog deleted on chromosome ten, epidermal growth factor receptor, complex, AKT confirming that EBP50 specifically mediated the formation of the PTEN/EGFR complex. Both PTEN (V403A) and EGFR (L1043/1063F) mutants can not bind with EBP50. The expression of PTEN (V403A) or EGFR (L1043/1063F) mutant in cells resulted in higher AKT activation level than their respective wild-types by EGF stimulation, indicating that EBP50-mediated PTEN/EGFR complex can effectively inhibit EGF-induced AKT activation. EGF stimulation of siEBP50 cells induced higher AKT activation level compared with control cells, further confirming EBP50-mediated PTEN/ EGFR complex can more effectively inhibit EGF-induced AKT activation. These results demonstrated the PTEN/EGFR complex formed under the mediation of EBP50, revealing a novel mechanism for negative regulation of EGF-induced AKT pathway, which may be an important molecular target for antineoplastic therapy.

\section{Introduction}

Epidermal growth factor receptor (EGFR) signaling is crucial for cell survival, growth, proliferation and migration $(1,2)$. Dysregulated EGFR signaling correlates with proliferation, invasiveness and drug resistance of multiple types of cancer, such as brain, lung, and breast cancer $(3,4)$. EGFR activation triggers cascades of multiple downstream signaling, including the phosphoinositide 3-kinase/AKT (PI3K/AKT) pathway, mitogen-activated protein kinase (MAPK) pathway and signal transducer and activator of transcription (STAT) pathway (2).

Among these pathways activated by EGFR, AKT signaling regulates many cellular functions including growth, survival, and invasiveness, its excessive activation plays important roles in cancer. EGFR-AKT signaling was involved in esophageal squamous cell carcinoma (ESCC) cell proliferation and migration (5). EGF-induced AKT signaling was also reported in ovarian cancer cell proliferation (6). PI3K/AKT signaling cascades may be responsible for EGF-activated MMP7 and consequent cancer invasiveness in human gastric carcinoma cell lines SNU-5 and HGC27 (7). In non-small cell lung cancer (NSCLC) cells EGF activated MMP9 via AKT 
signaling to promote NSCLC invasiveness (8). Activation of EGFR/PI3K/AKT pathway can also mediate hypoxia-induced drug resistance in hepatocellular carcinoma cells (9).

Inhibition of the EGF-induced EGFR/AKT activation was extensively used to promote cell apoptosis, suppress tumor cell growth and invasiveness. For example, in OVCAR-3 ovarian cancer cells, using PI3K inhibitors, but not ERK inhibitor, decreased EGF-induced cellular viability, revealing the important role of AKT pathway in EGF-induced cellular viability (6). AKT inhibitor significantly inhibited the EGF-induced activation of MMP7 in gastric cancer cells (7). PI3K inhibitor wortmannin inhibited the EGF-induced EGFR/PI3K/AKT signaling pathway concomitantly with inhibition of cell proliferation and cell cycle progression of primary external auditory canal keratinocytes (EACKs) (10).

Due to the important role of EGFR/AKT pathway activation in tumor cell malignant phenotype, regulation on EGFR/AKT pathway in vivo was studied by several groups (11-13). Phosphatases were reported as potent regulators of EGF-induced AKT signaling (13). Phosphatase PTPN2 (T-cell protein tyrosine phosphatase; also known as TC-PTP) inhibited EGF-induced AKT phosphorylation, but not ERK1/2 phosphorylation (12). Phosphatase PTPRJ (protein tyrosine phosphatase receptor type J; also known as DEP-1/CD148) was found to regulate EGF-induced PI3K and AKT activation (13). Among phosphatases, PTEN (phosphatase and tensin homologue deleted on chromosome 10), the most effective phosphatase, can also regulate EGF-induced AKT activation (13). However, how PTEN regulated EGF-induced AKT activation was not completely elucidated.

PTEN was found to inhibit the activation of the growth factor receptor PDGFR pathway, especially the activation of PDGF-induced AKT pathway (14). One of its mechanisms was via ternary complex PTEN/EBP50/PDGFR mediated by EBP50. EBP50 (Ezrin-radixin-moesin-binding phosphoprotein-50, also called NHERF) was reported to bind with PDGFR (15). EBP50 can also interact with PTEN $(14,16)$. So EBP50 could act as a bridge, recruit PTEN to PDGFR at plasma membrane (PM) and restrict the activation of the PI3K/AKT signaling. EBP50 expression also confers susceptibility to PDGFR pharmacological inhibition in breast cancer $(14,17)$. EBP50 mutations disrupting the PTEN/EBP50/PDGFR complex formation would inactivate the inhibition of EBP50 on PDGF-induced AKT activation. Both EBP50 K172N and D301V mutations found in breast cancer can abolish PTEN/EBP50/PDGFR complex formation, and retard the inhibition of PTEN on PDGF-induced AKT activation (18), revealing an emerging model regulating growth factor-mediated AKT signaling-adaptor protein bound with growth factor receptor and further mediated PTEN binding with growth factor receptor $(14,19)$.

Both our previous research results, and those of other groups revealed that EGFR can bind to EBP50 (20,21), and PTEN also interacted with EBP50 $(14,16)$, providing the rationale that EBP50 can organize the complex of PTEN and EGFR to regulate the EGFR/AKT pathway. Thus, in this study we first examined the formation of PTEN, EBP50 and EGFR complex by using co-immunoprecipitation (Co-IP) and GST pull-down assays. Further, we investigated the effect of their association on EGFR/AKT pathway by abolishing the complex formation. Results showed that EBP50 specifically scaffolds the interaction of PTEN with EGFR and enhances the inhibition of PTEN on EGF-induced AKT activation. These results elucidated a novel mechanism regulating EGF-induced AKT signaling.

\section{Materials and methods}

Preparation of plasmids. Glutathione S-transferase (GST)-tagged PTEN carboxyl-terminal (GST-PTEN-CT, encoding amino acid from 374 to 403) plasmid was generated via polymerase chain reaction (PCR) amplification of human PTEN cDNA, then inserted into pGEX-4T-1. The inserts were verified by DNA sequencing. Isopropyl $\beta$-D-1-Thiogalactopyranoside (IPTG) was used to induce the expression of fusion proteins.

pBK-CMV-hemagglutinin (HA)-EBP50 expression plasmid was kindly provided by Dr Randy Hall from Emory University (Atlanta, GA, USA). pSuper.puro EBP50 shRNA and pSuper.puro luciferase control shRNA plasmids were kindly provided by Dr Margaret J. Wheelock from University of Nebraska Medical Center (Omaha, NE, USA). Full-length PTEN was inserted into pBK-CMV-Flag vector for Flag-tagging. pBK-CMV-Flag-EGFR was kindly provided by Dr Howard A. Rockman from Duke University Medical Center (Durham, NC, USA).

PTEN carboxyl-terminal (CT) PDZ protein binding motif point mutation (V403A-the last amino acid V403 was mutated to A) and EGFR PDZ binding motif point mutation (L1043/1063F-key residue L1043 and L1063 of PDZ binding site were mutated to F) were created by PCR amplification and confirmed by sequencing.

Cell culture, transfection and cell treatments. African green monkey kidney cell line COS-7 and human astrocytoma U-373 MG (U373) cell lines (American Type Culture Collection, ATCC; Manassas, VA, USA) were grown in Dulbecco's modified Eagle's medium (DMEM; Gibco) and RPMI-1640 medium, respectively. Both media contained 10\% fetal bovine serum (FBS; Hyclone, Logan, UT, USA) and 1\% antibiotic-antimycotic agent (Life Technologies, Inc., Grand Island, NY, USA). Cells were grown at $37^{\circ} \mathrm{C}$ and $5 \% \mathrm{CO}_{2}$ to $80 \%$ confluency for use.

Transfections were performed by Lipofectamine 2000 (Invitrogen, Carlsbad, CA, USA) with plasmids DNA following the protocol as reported before (22).

COS-7 or U373 cells were serum starved overnight, then treated with $100 \mathrm{ng} / \mathrm{ml}$ EGF (Sigma-Aldrich, St. Louis, MO, USA) for different time periods at $37^{\circ} \mathrm{C}(20)$ to detect the effect of PDZ binding motif-mutated PTEN or EGFR overexpression on EGFR-mediated signal transduction pathway. The effect of EBP50 knockdown on EGFR-mediated signal transduction pathway was performed in the same way.

Stable transfection. For EBP50 stable knockdown, COS-7 cells were transfected with pSuper.puro EBP50 shRNA or control pSuper.puro luciferase shRNA plasmid, respectively using Hifectin II (Applygen Technologies Inc., Beijing, China) following the protocol. Two days following transfection, cells were transferred to $90-\mathrm{mm}$ plates and cultured in selection 
medium with $0.5 \mu \mathrm{g} / \mathrm{ml}$ puromycin (Sigma-Aldrich) to knock down EBP50 (20). The medium was changed every two days to remove floating dead cells, and the resistant colonies formed were harvested and plated in 24-well plates. Cell cultures were expanded and cultured for at least a month, then the fractions were used for analysis of EBP50 expression by western blotting, with GAPDH expression as a protein loading control. Stably-transfected cells were maintained and passaged in culture medium with puromycin $(0.25 \mu \mathrm{g} / \mathrm{ml})(20)$.

siRNA-mediated transient EBP50 knockdown. Small interfering RNA (siRNA) duplexes directed against EBP50 (nucleotides to: 5'-GUCGACCACCAGCAGGCGCACGGCG UUG-3') and control scrambled RNAi (5'-UCCAGACGGCG CAGUGGGCGACCGCUAC-3') were synthesized by Sigma-Aldrich. COS-7 cells were grown to $80 \%$ confluency in 35-mm dishes, transfected with $2 \mu 1$ Lipofectamine 2000 (Invitrogen), and mixed with 36 pmol of the synthetic EBP50 siRNA. The cells were then serum starved overnight, stimulated, harvested and analyzed after $48 \mathrm{~h}$ of transfection.

Western blotting. Samples were run on $8 \%$ sodium dodecyl sulfate (SDS)-polyacrylamide gels (PAGE) and transferred to PVDF membranes. The blots were blocked in blocking buffer (5\% non-fat dry milk in TBST buffer) for $1 \mathrm{~h}$ at room temperature and then incubated with primary antibodies in blocking buffer overnight at $4^{\circ} \mathrm{C}$. The blots were washed three times with TBST buffer and incubated for $1 \mathrm{~h}$ at room temperature with a horseradish peroxidase (HRP)-conjugated anti-mouse $\mathrm{IgG}$ and anti-rabbit IgG secondary antibody (Amersham Biosciences, Piscataway, NJ, USA) in blocking buffer. Finally, the blots were washed three times with TBST buffer and visualized via enzyme-linked chemiluminescence using the electrochemiluminescence (ECL) kit (Applygen Technologies Inc.) (23). The results of western blotting were semi-quantitatively analyzed by ImageJ software (NIH, Bethesda, MD, USA).

Protein levels were normalized with GAPDH, and the levels of phospho-AKT immunoreactivity were normalized to the total AKT immunoreactivity. The primary antibody specific for the EBP50 was purchased from BD Biosciences (San Jose, CA, USA), anti-HA was from MBL (Nagoya, Japan), anti-Flag antibody was from Sigma-Aldrich. Other primary antibodies specific for GAPDH, PTEN, phospho-AKT (Ser473), total AKT were all bought from Cell Signaling Technology (Beverly, MA, USA).

GST pull-down assay. GST fusion proteins were purified from bacteria using glutathione-sepharose 4B beads (Sigma-Aldrich) according to the manufacturer's protocol. The GST pull-down assay was performed as described previously (24). Briefly, equal amounts of GST or GST-PTEN-CT (amino acid from 374 to 403, WT or V403A mutant-the last amino acid V403 mutated to $\mathrm{A}$ ) fusion protein beads were incubated with equal amounts of cell lysates. After incubation at $4^{\circ} \mathrm{C}$ for $2 \mathrm{~h}$, the beads were washed with ice-cold wash buffer. Proteins were then eluted with SDS sample buffer, and detected with western blotting.

Co-immunoprecipitation assay. Co-immunoprecipitation (Co-IP) was performed as described (25). Briefly, the rabbit kidney tissues were homogenized in ice-cold lysis buffer

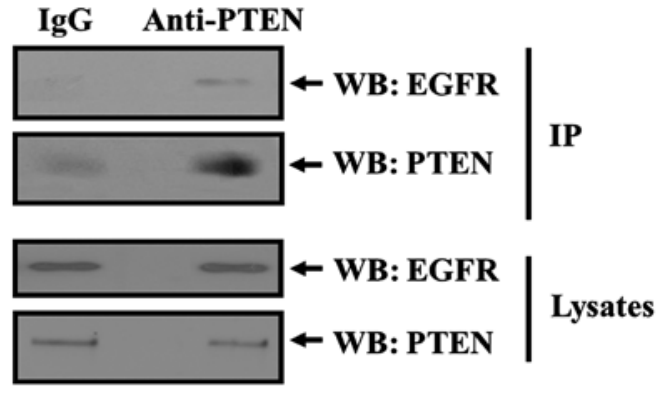

Figure 1. PTEN interacted with EGFR in rabbit kidney tissues. Solubilized lysates from homogenized rabbit kidney tissues were subjected to immunoprecipitation with a specific anti-PTEN antibody. The immunoprecipitated complexes were then probed with an anti-EGFR antibody via western blotting. Co-immunoprecipitation of EGFR with PTEN from tissue, but no co-immunoprecipitation of IgG with PTEN was observed.

(10 mmol/1 HEPES, $50 \mathrm{mmol} / 1 \mathrm{NaCl}, 5 \mathrm{mmol} / 1$ EDTA, $1 \mathrm{mmol} / \mathrm{l}$ benzamidine, $0.5 \%$ Triton $\mathrm{X}-100, \mathrm{pH}$ 7.4). Lysates were solubilized and clarified. Supernatants were incubated with anti-PTEN antibody (Cell Signaling Technology), prebounded with protein A\&G beads (Calbiochem, San Diego, CA, USA). After washing with an ice-cold lysis buffer three times, the immunoprecipitated proteins were eluted from the beads with SDS sample loading buffer. The eluted samples were then analyzed by western blotting. Anti-PTEN and antiEGFR antibody were used in this study.

Statistical analyses. All data are presented as means \pm SD and statistical significance was analyzed by ANOVA. Differences were considered significant at $\mathrm{P}<0.05$.

\section{Results}

PTEN forms a complex with EGFR in tissues. In order to verify that PTEN formed a complex with EGFR, we chose rabbit kidney tissues which express high level of PTEN, EGFR and EBP50 to perform Co-IP study. Solubilized lysates from rabbit kidney tissues were incubated with $\mathrm{IgG}$ or PTEN antibody linked to protein A/G-agarose beads. Co-precipitated EGFR was detected by western blotting with anti-EGFR antibody. Co-immunoprecipitation of EGFR with PTEN in rabbit kidney tissues was detected, whereas no co-immunoprecipitation of IgG with PTEN was observed (Fig. 1). Co-IP assay result of tissue showed that PTEN could co-immunoprecipate with EGFR and provided evidence of a physical complex between PTEN and EGFR in tissues.

EBP50 protein mediates the formation of the PTEN/EGFR complex. To further clarify whether EGFR forms a complex with PTEN via their direct interaction, or via adaptor protein EBP50, we detected the formation of PTEN/EGFR complex in the absence of EBP50. COS-7 cells were transfected with the pSuper.puro-EBP50 shRNA plasmid or the control plasmid, respectively. The EBP50 stable knockdown cell line (shEBP) and its control cell line (shLuc) were generated by puromycin screening. Verification of protein knockdown was determined by western blot analysis as shown in Fig. 2A. In shLuc cells, EBP50 expression level was the same as that in 
A
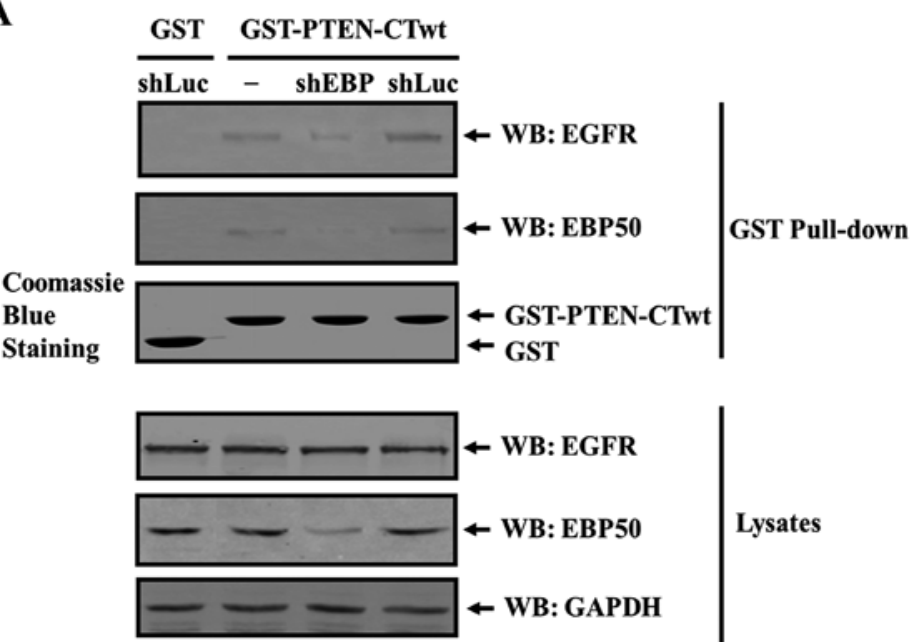

B

HA-EBP50

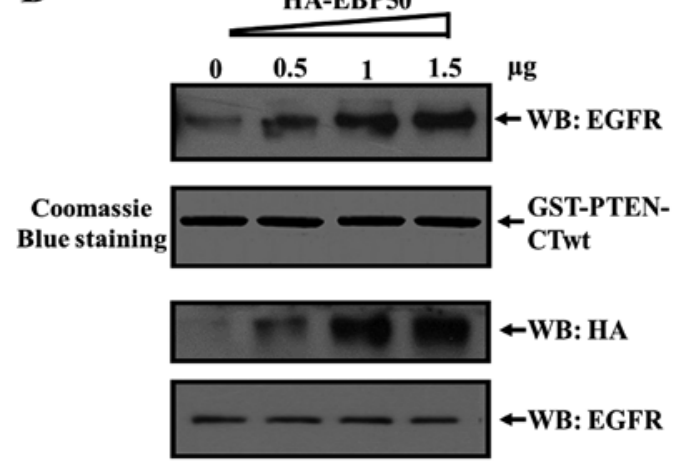

GST Pull-down

C

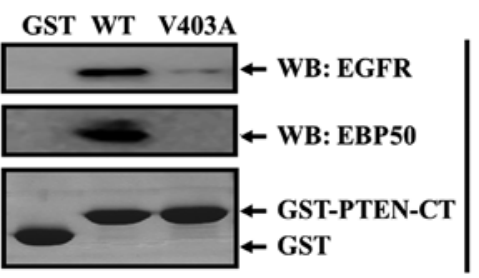

GST Pull-down

Coomassie Blue staining

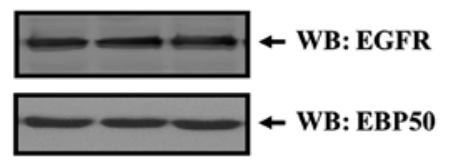

Lysates

Figure 2. EBP50 protein specifically facilitated the formation of PTEN/EBP50/EGFR complex. (A) The knockdown of EBP50 disrupted the ternary complex formation. EBP50 expression in COS-7 cells was knocked down with pSuper.puro-EBP50 plasmid or control pSuper.puro-Luciferase plasmid and the cells were called as shEBP and shLuc cells, respectively. With the knockdown of EBP50, the EGFR pull-down by PTEN-CTwt was almost abolished, but luciferase knockdown did not abolish the interaction between EGFR and PTEN. (B) EBP50 expression increased the amount of EGFR pulled down by GST-PTEN-CTwt in a dose dependent manner. With the increase of EBP50 expression level, the EGFR pull-down by PTEN-CTwt was increased accordingly. Coomassie blue staining revealed equal loading of the fusion proteins. (C) PDZ binding motif mutant PTEN did not interact with EGFR. The PTEN-CTwt interacted with EBP50 and EGFR, but not PTEN-CT (V403A)mt. Coomassie blue staining revealed equal loading of the fusion proteins.

parental cells. In shEBP cells, EBP50 expression was stably knocked down up to $67 \%$ compared to that of its parental cells. Subsequently, these cell lysates were collected and subjected to GST pull-down assay. As shown in Fig. 2A, EGFR and EBP50 signals were detected from the GST-PTEN-CT pulldown complex in COS-7 parental cells and shLuc cells, but not in shEBP cells, indicating PTEN/EGFR complex formation was mediated by EBP50 and the knockdown of EBP50 disrupted the ternary complex formation.

EBP50 knockdown disrupted the formation of the PTEN/EGFR complex, preliminarily suggesting that EBP50 can act as an adaptor to assemble the PTEN/EGFR complex. Thus, it is possible that the increase of EBP50 expression level will lead to an increase in the complex amount. We further detected whether PTEN/EGFR complex was facilitated in EBP50-concentration dependent manner. We transiently transfected increasing dose of pBK-CMV-HA-EBP50 expression plasmid (0, 0.5, 1 and $1.5 \mu \mathrm{g}$, respectively) into COS-7 cells, then collected the lysates of these cells to perform GST pulldown experiment and to detect EGFR in the GST pull-down fraction. Results showed that in cell lysates the expression level of exogenous EBP50 protein increased. Whereas, EGFR 
A

\begin{tabular}{|c|c|c|}
\hline \multicolumn{3}{|c|}{ COS-7 } \\
\hline vector & $\begin{array}{c}\text { PTEN } \\
\text { (wt) }\end{array}$ & $\begin{array}{c}\text { PTEN } \\
(\text { V403A) }\end{array}$ \\
\hline 30 & 0 & 30 \\
\hline
\end{tabular}

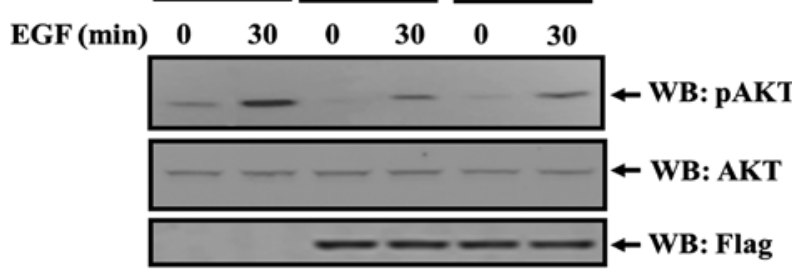

B

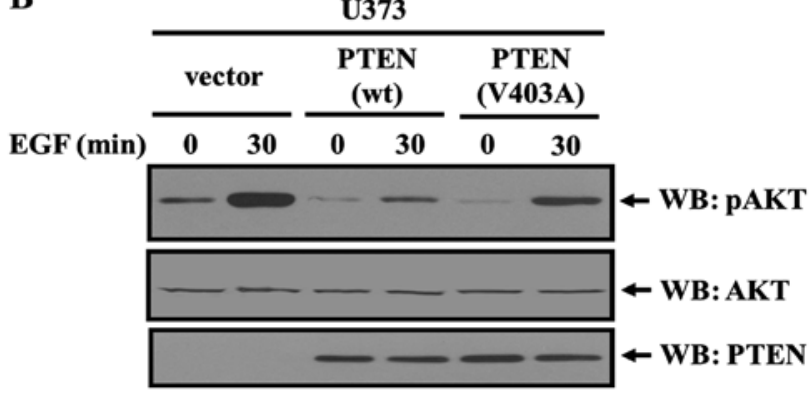

Figure 3. The PTEN/EBP50/EGFR complex attenuated EGF-induced AKT signaling. (A) PDZ binding motif mutant PTEN enhances EGF-induced AKT activation and prevents inhibition by EBP50. The data presented are representative of a minimum of three independent experiments. (B) PTEN-fl (wt) which can form a ternary complex suppressed the EGF-induced AKT activation, but PTEN-fl (V403A) did not. In PTEN-loss U373 cells, PDZ binding motif mutant PTEN enhances EGF-induced AKT activation level and prevents inhibition by EBP50 more obviously. The data presented are representative of a minimum of three independent experiments. Again, PTEN-fl (wt) which can form a ternary complex suppressed the EGFRmediated AKT signaling, but PTEN-fl (V403A) did not.

expression level was not influenced. When these cell lysates were pulled down by GST-PTEN-CTwt fusion protein, with the increase of EBP50 expression level, the amount of EGFR pulled down by GST-PTEN-CTwt increased accordingly (Fig. 2B), further verifying that EBP50 mediated the formation of PTEN/EGFR complex.

EBP50 bound with PTEN and EGFR via their PDZ binding motifs. When PDZ binding motif of PTEN or EGFR is mutated (PTEN V403A, EGFR L1043/1063F) and can not bind with EBP50, the PTEN/EGFR complex may not form. To confirm this hypothesis, we expressed GST-PTEN-CT wild-type (wt) and V403A mutant fusion proteins, respectively, and used them to pull down COS-7 cell lysates. As shown in Fig. 2C, GST-PTEN-CT-wt can pull down EBP50 and EGFR from COS-7 lysates, whereas GST and GST-PTEN-CT-mt (V403A) can not. This suggested that EGFR did not associate with PTEN when EBP50 could not bind with PTEN, indicating that EBP50 specifically mediated the formation of PTEN/EGFR complex.

Taken together,EBP50 mediated the PTEN/EGFR complex formation and the adaptor role of EBP50 as PTEN/EGFR complex is specific.

PTEN binding with EGFR regulates EGFR/AKT signaling. PTEN/EBP50/PDGFR complex regulated PDGF-induced AKT signaling (14), and the PTEN/EBP50/EGFR complex may also regulate EGF-induced AKT signaling. We next assessed the regulatory roles of EBP50 and PTEN in EGF-induced
AKT activation. Flag control vector, flag-PTEN-WT, flagPTEN-V403A mutant was transiently transfected into COS-7 cells, respectively, then EGF stimulation was performed for 30 min to observe the EGF-induced AKT activation level in these transfected cells. In PTEN-WT transfected cells, AKT activity by EGF stimulation was lower than that in vector control cells (only $34 \%$ of that in vector control cells). The AKT activation level in PTEN-V403A transfected cells was relatively higher than that in PTEN-WT transfected cells $(60 \%$ of that in vector control cells, $\mathrm{P}<0.05$, Fig. $3 \mathrm{~A}$ ).

Interestingly, similar result was revealed in U373 cells which have no endogenous PTEN expression. In PTEN-WT transfected U373 cells, AKT activity by EGF stimulation was lower than that in vector control cells (only $23 \%$ of that in vector control cells), but in PTEN-V403A transfected U373 cells AKT activation level was relatively higher than that in PTEN-WT transfected U373 cells (54\% of that in vector control cells) ( $\mathrm{P}<0.05$, Fig. 3B). As PTEN-V403A mutant fails to interact with EBP50, it is unable to mediate the formation of PTEN/EBP50/EGFR trimer. This result indicated that more active AKT in PTEN-V403A transfected cells compared with PTEN-WT transfected cells may result from the failure of PTEN/EBP50/EGFR trimer formation, and consequent lack of ability of PTEN in inhibiting EGF-induced AKT pathway.

EGFR L1043/1063F mutant can not bind with EBP50 (21). To further confirm the role of the EBP50-mediated PTEN/ EGFR complex, flag control vector, flag-EGFR-WT, flagEGFR-L1043/1063F mutant which can not bind to EBP50 was transiently transfected into COS-7 cells, respectively. Then EGF stimulation was performed for $30 \mathrm{~min}$ to observe the activation level of AKT in these transfected cells. Results showed that, in EGFR-WT transfected cells, AKT activation level by EGF stimulation was higher than that in vector control cells (1.43-fold over that in vector control cells). However, AKT activation in EGFR-L1043/1063F transfected cells was relatively higher than that in vector control cells, even higher than that in EGFR-WT transfected cells (2.22-fold over that in vector control cells and 1.56-fold over that in EGFR-WT transfected cells, respectively) ( $\mathrm{P}<0.05$, Fig. 4A). As EGFR-L1043/1063F mutant can not interact with EBP50, it is unable to mediate the formation of PTEN/EBP50/EGFR trimer. This result further indicated that it was the failure of the trimer formation that caused the failure of PTEN in inhibiting EGFR-AKT pathway and more active AKT in EGFR-L1043/1063F mutant transfected cells than EGFR-WT transfected cells.

Consistent with these results, disruption of this complex led to an increase of EGF-induced AKT activation level. EBP50 siRNA was used to knock down the expression of EBP50 in COS-7 cells, its EBP50 expression was knocked down by $\sim 52 \%$, and its AKT activation level was 1.57- and 1.62-fold over that in scrambled siRNA transfected cells at 15 and $30 \mathrm{~min}$ of EGF stimulation, respectively ( $\mathrm{P}<0.05$, Fig. $4 \mathrm{~B}$ ), revealing that EBP50 knockdown led to stronger AKT activation. Thus, these results consistently demonstrate that the ternary complex between PTEN, EBP50 and EGFR leads to a specific inhibition role for EGF-induced AKT activation.

Altogether, these results clearly reveal that EBP50-mediated PTEN/EGFR complex can inhibit EGF-induced EGFR/AKT pathway activation more effectively. 
A
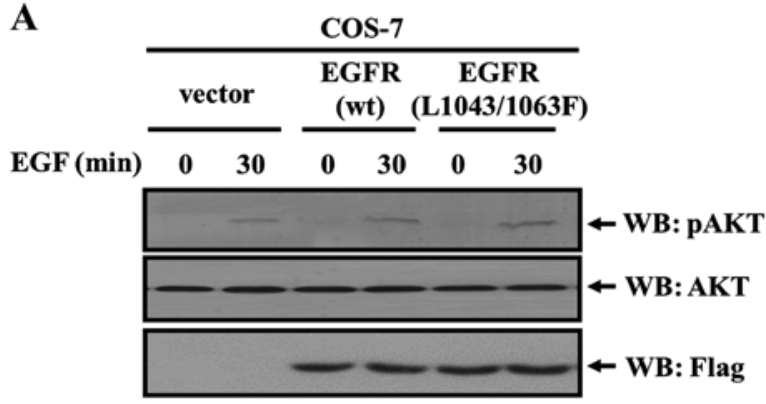

B

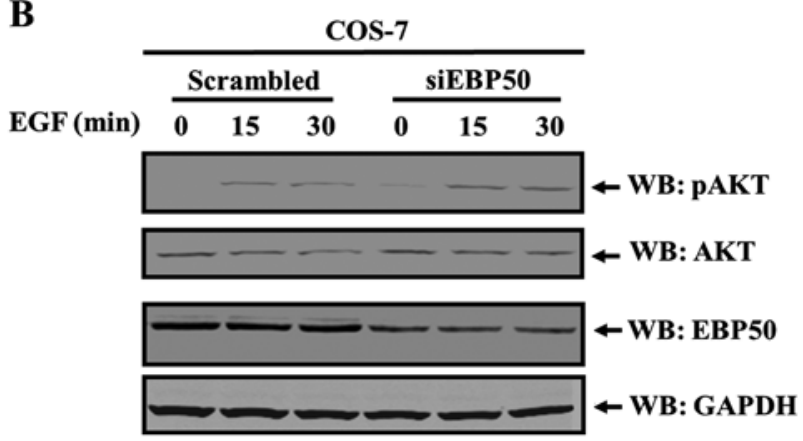

Figure 4. The PTEN/EBP50/EGFR complex attenuated EGF-induced AKT signaling. (A) PDZ binding motif mutant EGFR enhances EGF-induced AKT activation level and prevents inhibition by EBP50. Although EGFR-fl (wt) which can form ternary complex promoted the EGF-induced AKT signaling, EGFR-fl (L1043/1063F) promoted EGF-induced AKT signaling more strongly, suggesting the complex formation can suppress EGF-induced AKT signaling. (B) Disruption of the complex by EBP50 knockdown promoted EGF-induced AKT phosphorylation. EGF-stimulated AKT phosphorylation in siEBP50 cells was significantly higher than that in control cells. The data presented are representative of a minimum of three independent experiments.

\section{Discussion}

In this study, we found that EBP50 mediated EGFR/PTEN super-macromolecular complexation and inhibited the activation of EGF-induced AKT signaling pathway. Mutating either EGFR (L1043/1063F) or PTEN (V403A) to abolish its binding with EBP50, severely interrupted the formation of PTEN/EGFR complex, and knockdown of EBP50 expression in cells also abolished the PTEN/EGFR complex. Overexpressing EGFR (L1043/1063F) or PTEN (V403A) mutants, which can not form PTEN/EGFR complex, in cells enhanced the phosphorylation level of EGF-induced AKT. Consistently, EBP50 knockdown in cells also enhanced the phosphorylation level of EGF-induced AKT. These results demonstrated that EBP50 as a scaffold organized the assembly of PTEN/EGFR complex and mediated the inhibition of AKT activation induced by EGF. This finding reveals a new macromolecular signaling complex involving in EGFR and helps to better understand the regulatory mechanism of EGFR signaling pathway.

PTEN phosphatase domain is located in its N-terminal (NT) and PTEN-NT missense mutant lost its phosphatase activity $(26,27)$. Interestingly, PTEN C-terminal (CT, codons 212-403) missense mutants were also reported to have relatively low phosphatase activity (28-31). In this study we found that PTEN-CT V403A mutant also showed low phosphatase activity for pAKT (Fig. 3). This phenomenon remains a mystery. We found PTEN-CT was very important in binding with EBP50 and forming a complex with EGFR. This complex could attenuate EGF-induced AKT activity, providing the additional mechanism that PTEN CT mutant weakens its ability to suppress EGF-induced AKT activation.

In clinical samples, some EGFR mutation sites [T790M (32), L858R (33), L861Q (34)] were not in the autophosphorylation site of EGFR, but their signaling activation ability was stronger than that of EGFR WT. Some EGFR single nucleotide polymorphisms [SNPs, R962G, R977C, H988P (35)] were not in the autophosphorylation site of EGFR, but their signaling activation ability was also stronger than that of EGFR WT. Its molecular mechanism remains unknown. These EGFR mutations or SNPs are close to theEBP50 interacting site (1037-1065), it is possible that the stronger EGFR signaling activation results from the failure in forming the EGFR/EBP50/PTEN complex and further failure in inhibiting EGF-induced AKT signaling. In this study, EGFR mutant (L1043/1063F) expression also triggered stronger EGFR-mediated AKT signaling. Its mechanism was involved in failure in forming the EGFR/ EBP50/PTEN complex, supporting the important clinical significance of EBP50 binding in EGFR signaling regulation. However, this needs to be further explored.

As an adaptor protein, EBP50 plays an important role in the formation of the EGFR/EBP50/PTEN complex. The change of EBP50 expression level possibly influence the formation of the complex, further influencing the activation of AKT pathway. Thus, we performed data mining from the public database NCBIs Gene Expression Omnibus (GEO, http://www.ncbi.nlm.nih.gov/geo/) Profile (GSE6344 data from clear-cell renal cell carcinoma cases) and selected samples with similar EGFR, PTEN and $A K T$ gene expression levels between cancer and paracancer tissues $(\mathrm{P}>0.05$ according to paired-sample t-test paracancer samples vs. cancer samples. EGFR $201.64 \pm 68.23$ vs. $199.13 \pm 67.54$; PTEN $24.93 \pm 21.99$ vs. $22.56 \pm 25.71$, AKT $592.72 \pm 87.42$ vs. $731.94 \pm 153.48)$. We then examined the correlation between expression levels of EBP50 and gene sets involved in EGFR and AKT pathway in clinical samples by Gene set enrichment analysis (GSEA; http://www. broad.Mit.edu/gsea). The expression level of a priory defined positive EGFR gene set (GNF2_EGFR) and AKT pathway gene set (PID_PI3KCI_AKT_pathway and KEGG_mTOR _ Signaling_pathway, c2. v4.0 from the Molecular Signatures Database-MsigDB, http://www.broad.mit.edu/gsea/msigdb/ index.jsp) were used as an indicator of the activation of the EGFR pathway and AKT pathway, respectively. GSEA result showed that both their EGFR gene set and AKT gene set were enriched in EBP50 lower expression patients, respectively, suggesting that EBP50 expression negatively contributed to EGFR and AKT gene set expression in these patients ( $F D R=0.002, P=0.001$ and $F D R=0.03, P=0.02$, respectively). GSEA result partly verified EBP50 retarded EGF-induced AKT signaling from clinical data, also supporting that EGFR/ EBP50/PTEN complex plays an important regulatory role in EGFR and AKT signaling pathway.

In addition, in some cervical clear cell carcinoma cases, AKT phosphorylation level increased (36). Interestingly, the expression level of EGFR which can increase AKT phosphorylation level did not significantly increase (from the human protein atlas result) or EGFR was even expressed 
in low level (data analysis results of Pubmed GEO Profile cervical carcinoma data GDS3233), reminding that the increase of AKT phosphorylation level may not completely result from the increase of EGFR expression level. EBP50 expression level was significantly downregulated (from the Human Protein Atlas result and GDS3233 data, paracancer samples $10.06 \pm 0.65$ vs. cancer samples $9.07 \pm 0.88, \mathrm{P}<0.01$, independent-samples t-test). Because this study demonstrated that EBP50 mediated the formation of EGFR/PTEN complex, it is possible that decreased EBP50 expression in this type of tumor may explain the pathogenesis and further elucidate the importance of the PTEN-EBP50 connection for cancer progression. However, this needs to be further experimentally verified.

EBP50 may behave either as a tumor suppressor, when it is localized at the plasma membrane (PM), or as an oncogenic protein, when it is at the cytoplasm and nuclei $(37,38)$. Its molecular mechanism remains unclear. Current results showed that only when EBP50 bound with EGFR localized in PM, it can recruit PTEN and form a complex with EGFR, then regulate EGF-induced AKT phosphorylation. When EBP50 did not bind with EGFR localized at PM, it can not mediate the EGFR/PTEN complex formation and regulate EGF-induced AKT signaling. Binding with EGFR in PM may be one of the mechanisms for EBP50 to be a tumor suppressor.

In summary, this study clarified the nature of EGFR/AKT pathway regulation by PTEN under the mediation of EBP50, and demonstrated an additional level of extrinsic protein regulation through binding partner interactions. EBP50 and PTEN proteins suppress EGF-induced AKT signaling and points to a helper role for EBP50 proteins in PTEN tumor suppressor activity.

\section{Acknowledgements}

This study was supported by the National Natural Science Foundation of the People's Republic of China (no. 30900247 and 81372739), the Importation and Development of HighCaliber Talents Project of Beijing Municipal Institutions (CIT\&TCD201304187) and Beijing Municipal Science and Technology Commission (no. Z151100001615039).

\section{References}

1. Safdari Y, Khalili M, Ebrahimzadeh MA, Yazdani Y and Farajnia S: Natural inhibitors of PI3K/AKT signaling in breast cancer: Emphasis on newly-discovered molecular mechanisms of action. Pharmacol Res 93: 1-10, 2015.

2. Jones S and Rappoport JZ: Interdependent epidermal growth factor receptor signalling and trafficking. Int J Biochem Cell Biol 51: 23-28, 2014.

3. Pan D and Lin X: Epithelial growth factor receptor-activated nuclear factor $\mathrm{\kappa B}$ signaling and its role in epithelial growth factor receptor-associated tumors. Cancer J 19: 461-467, 2013.

4. Han W and Lo HW: Landscape of EGFR signaling network in human cancers: Biology and therapeutic response in relation to receptor subcellular locations. Cancer Lett 318: 124-134, 2012.

5. Liu R, Gu J, Jiang P, Zheng Y, Liu X, Jiang X, Huang E, Xiong S, $\mathrm{Xu}$ F, Liu G, et al: DNMT1-microRNA126 epigenetic circuit contributes to esophageal squamous cell carcinoma growth via ADAM9-EGFR-AKT signaling. Clin Cancer Res 21: 854-863, 2015.

6. Khabele D, Kabir SM, Dong Y, Lee E, Rice VM and Son DS: Preferential effect of akt2-dependent signaling on the cellular viability of ovarian cancer cells in response to EGF. J Cancer 5: 670-678, 2014.
7. Liu G, Jiang C, Li D, Wang R and Wang W: miRNA-34a inhibits EGFR-signaling-dependent MMP7 activation in gastric cancer. Tumour Biol 35: 9801-9806, 2014.

8. Pei J, Lou Y, Zhong R and Han B: MMP9 activation triggered by epidermal growth factor induced FoxO1 nuclear exclusion in non-small cell lung cancer. Tumour Biol 35: 6673-6678, 2014.

9. Wang XJ, Feng CW and Li M: ADAM17 mediates hypoxia-induced drug resistance in hepatocellular carcinoma cells through activation of EGFR/PI3K/Akt pathway. Mol Cell Biochem 380: 57-66, 2013.

10. Liu W, Ren H, Ren J, Yin T, Hu B, Xie S, Dai Y, Wu W, Xiao Z, Yang X, et al: The role of EGFR/PI3K/Akt/cyclinD1 signaling pathway in acquired middle ear cholesteatoma. Mediators Inflamm 2013: 651207, 2013.

11. Tiganis T, Kemp BE and Tonks NK: The protein-tyrosine phosphatase TCPTP regulates epidermal growth factor receptor-mediated and phosphatidylinositol 3-kinase-dependent signaling. J Biol Chem 274: 27768-27775, 1999.

12. Scharl M, Rudenko I and McCole DF: Loss of protein tyrosine phosphatase $\mathrm{N} 2$ potentiates epidermal growth factor suppression of intestinal epithelial chloride secretion. Am J Physiol Gastrointest Liver Physiol 299: G935-G945, 2010.

13. Omerovic J, Clague MJ and Prior IA: Phosphatome profiling reveals PTPN2, PTPRJ and PTEN as potent negative regulators of PKB/Akt activation in Ras-mutated cancer cells. Biochem J 426: 65-72, 2010.

14. Takahashi Y, Morales FC, Kreimann EL and Georgescu MM: PTEN tumor suppressor associates with NHERF proteins to attenuate PDGF receptor signaling. EMBO J 25: 910-920, 2006.

15. Maudsley S, Zamah AM, Rahman N, Blitzer JT, Luttrell LM, Lefkowitz RJ and Hall RA: Platelet-derived growth factor receptor association with $\mathrm{Na}(+) / \mathrm{H}(+)$ exchanger regulatory factor potentiates receptor activity. Mol Cell Biol 20: 8352-8363, 2000.

16. Yang L, Wang Y, Chen P, Hu J, Xiong Y, Feng D, Liu H, Zhang H, Yang $\mathrm{H}$ and $\mathrm{He} \mathrm{J}: \mathrm{Na}(+) / \mathrm{H}(+)$ exchanger regulatory factor 1 (NHERF1) is required for the estradiol-dependent increase of phosphatase and tensin homolog (PTEN) protein expression. Endocrinology 152: 4537-4549, 2011.

17. Pan Y, Weinman EJ and Dai JL: $\mathrm{Na}^{+} / \mathrm{H}^{+}$exchanger regulatory factor 1 inhibits platelet-derived growth factor signaling in breast cancer cells. Breast Cancer Res 10: R5, 2008.

18. Cheng S, Li Y, Yang Y, Feng D, Yang L, Ma Q, Zheng S, Meng R, Wang S, Wang S, et al: Breast cancer-derived K172N, D301V mutations abolish $\mathrm{Na}^{+} / \mathrm{H}^{+}$exchanger regulatory factor 1 inhibition of platelet-derived growth factor receptor signaling. FEBS Lett 587: 3289-3295, 2013.

19. Rodriguez S and Huynh-Do U: Phosphatase and tensin homolog regulates stability and activity of EphB1 receptor. FASEB J 27: 632-644, 2013.

20. Yao W, Feng D, Bian W, Yang L, Li Y, Yang Z, Xiong Y, Zheng J, Zhai $\mathrm{R}$ and He J: EBP50 inhibits EGF-induced breast cancer cell proliferation by blocking EGFR phosphorylation. Amino Acids 43: 2027-2035, 2012.

21. Lazar CS, Cresson CM, Lauffenburger DA and Gill GN: The $\mathrm{Na}^{+} / \mathrm{H}^{+}$exchanger regulatory factor stabilizes epidermal growth factor receptors at the cell surface. Mol Biol Cell 15: 5470-5480, 2004.

22. Zheng J, Shen H, Xiong Y, Yang X and He J: The betal-adrenergic receptor mediates extracellular signal-regulated kinase activation via Galphas. Amino Acids 38: 75-84, 2010.

23. Zheng JF, Sun LC, Liu H, Huang Y, Li Y and He J: EBP50 exerts tumor suppressor activity by promoting cell apoptosis and retarding extracellular signal-regulated kinase activity. Amino Acids 38: 1261-1268, 2010.

24. Sun C, Zheng J, Cheng S, Feng D and He J: EBP50 phosphorylation by $\mathrm{Cdc} 2 /$ cyclin $\mathrm{B}$ kinase affects actin cytoskeleton reorganization and regulates functions of human breast cancer cell line MDA-MB-231. Mol Cells 36: 47-54, 2013.

25. Yang X, Zheng J, Xiong Y, Shen H, Sun L, Huang Y, Sun C, $\mathrm{Li}$ Y and He J: Beta-2 adrenergic receptor mediated ERK activation is regulated by interaction with MAGI-3. FEBS Lett 584: 2207-2212, 2010

26. He X, Arrotta N, Radhakrishnan D, Wang Y, Romigh T and Eng C: Cowden syndrome-related mutations in PTEN associate with enhanced proteasome activity. Cancer Res 73: 3029-3040, 2013.

27. Xu J, Li Z, Wang J, Chen H and Fang JY: Combined PTEN mutation and protein expression associate with overall and disease-free survival of glioblastoma patients. Transl Oncol 7: 196-205.e1, 2014 
28. Zhang SJ, Endo S, Ichikawa T, Yoshimura J, Onda K, Tanaka R, Washiyama $\mathrm{K}$ and Kumanishi T: Rare-type mutations of MMAC1 tumor suppressor gene in human glioma cell lines and their tumors of origin. Jpn J Cancer Res 90: 934-941, 1999.

29. Valiente M, Andrés-Pons A, Gomar B, Torres J, Gil A, Tapparel C, Antonarakis SE and Pulido R: Binding of PTEN to specific PDZ domains contributes to PTEN protein stability and phosphorylation by microtubule-associated serine/threonine kinases. J Biol Chem 280: 28936-28943, 2005.

30. Xu D, Yao Y, Jiang X, Lu L and Dai W: Regulation of PTEN stability and activity by Plk3. J Biol Chem 285: 39935-39942, 2010.

31. Maxwell GL, Risinger JI, Gumbs C, Shaw H, Bentley RC Barrett JC, Berchuck A and Futreal PA: Mutation of the PTEN tumor suppressor gene in endometrial hyperplasias. Cancer Res 58: 2500-2503, 1998

32. Barton S, Starling N and Swanton C: Predictive molecular markers of response to epidermal growth factor receptor (EGFR) familytargeted therapies. Curr Cancer Drug Targets 10: 799-812, 2010.

33. Suzuki T, Fujii A, Ohya J, Amano Y, Kitano Y, Abe D and Nakamura H: Pharmacological characterization of MP-412 (AV-412), a dual epidermal growth factor receptor and ErbB2 tyrosine kinase inhibitor. Cancer Sci 98: 1977-1984, 2007.
34. Yang S, Qu S, Perez-Tores M, Sawai A, Rosen N, Solit DB and Arteaga CL: Association with HSP90 inhibits Cbl-mediated down-regulation of mutant epidermal growth factor receptors. Cancer Res 66: 6990-6997, 2006.

35. Choura M,Frikha F, Kharrat N, Aifa S and Rebaï A: Investigating the function of three non-synonymous SNPs in EGFR gene: Structural modelling and association with breast cancer. Protein J 29: 50-54, 2010.

36. Ueno S, Sudo T, Oka N, Wakahashi S, Yamaguchi S, Fujiwara K, Mikami Y and Nishimura R: Absence of human papillomavirus infection and activation of PI3K-AKT pathway in cervical clear cell carcinoma. Int J Gynecol Cancer 23: 1084-1091, 2013.

37. Georgescu MM, Morales FC, Molina JR and Hayashi Y: Roles of NHERF1/EBP50 in cancer. Curr Mol Med 8: 459-468, 2008.

38. Shibata T, Chuma M, Kokubu A, Sakamoto M and Hirohashi S: EBP50, a beta-catenin-associating protein, enhances Wnt signaling and is over-expressed in hepatocellular carcinoma. Hepatology 38: 178-186, 2003. 